\title{
A Comprehensive Guide to Fuel Treatment Management Practices for Dry Mixed Conifer Forests in the Northwestern United States
}

USDA

United States Department of Agriculture

Forest Service

Rocky Mountain Research Station

Research Note RMRS-RN-63

April 2014

\section{Monitoring}

Short- and medium-term evaluation of how fuel treatments are working is the only way to know if the hundreds of activities on the ground are adding up to the goals of more resilient landscapes and increased safety of people and property. Monitoring is a critical resource for decision makers who design fuels management programs, however it is an often neglected part of the fuel management cycle.

A commitment to monitoring can lead to more effective and efficient fuels management programs. Clear and focused monitoring objectives ensure that investments in monitoring produce useful and timely information that can improve the effectiveness and efficiency of fuels management efforts.

Monitoring information can be used to:

- Review the efficacy and cost effectiveness of a treatment program or identify beneficial modifications to particular prescriptions.

- Effectively communicate with stakeholders and resource staff about desired forest conditions resulting from thoughtfully designed management.

- Establish a baseline against which treated stands can be compared when assessing forest conditions before and after treatment.

- Provide information on what is likely to happen when wildfire moves into a treated stand.

- Adequate monitoring can also help answer questions such as: How do the parameters for a prescribed fire (for example, duff moister content and wind speed) influence fuel consumption for a particular kind of forest structure and topographic setting?

\section{Developing a Monitoring Plan}

Using targeted questions to guide monitoring can aid in formulating a customized, relevant, concise and achievable monitoring plan. By focusing on addressing a few key questions, it ensures that the right information is collected to efficiently address specific objectives. Jain and others (2012) provide detail concerning each of these questions that can be used to develop an effective monitoring plan.

1. What are the project objectives and goals?

2. How will the monitoring information be used to adapt future prescriptions so that they are more successful? How will monitoring information obtained after a disturbance such as a wildfire be used to guide future management activities?

3. What will be monitored?

4. How much will monitoring cost, and who will pay?

5. How will monitoring be accomplished?

6. Who will do the monitoring?

7. When and where does the monitoring need to occur?

8. Where will monitoring data be stored, archived, and documented?

9. How, when, and by whom will the monitoring data be analyzed? 


\section{Monitoring Phases}

There are essentially three phases of fuels management outcomes: short term, medium-and long-term. Short-term monitoring addresses the immediate results of a treatment in terms of the properties of the stand it leaves behind, while longer-term monitoring can provide information about the effectiveness of fuel treatments to reduce the incidence of large fires by providing suppression opportunities or enhance forest resiliency.

Short-term Monitoring: Shortly after a fuel treatment, decision makers and forest managers may want to know how a fuel treatment performed at a local or stand scale. Questions may include:

- Did the project meet the objectives such as:

o Were surface fuels altered sufficiently to meet the objectives?

o Was canopy base height elevated to the level called for in the objectives?

o Were ladder fuels removed?

Medium- and Long-term Monitoring: Although the full benefits of longer-term monitoring may be years down the road, it is a critical component of fuel management. Monitoring questions may ask: How did fuel treatments perform when there is a fire? Do they slow fire growth sufficiently to make large fires less common? Do they change fire behavior in a way that reduces threats to people and property? Longer term questions include: Which treatments produce forests that are more resilient to fire and other disturbance agents?
How do treatments affect carbon storage and emissions, with and without subsequent wildfire?

Non-statistical surveys are no substitute for thoughtful, carefully planned monitoring. Although anecdotal evidence can provide some useful information, the full power of monitoring data requires a sizable store of statistically representative monitoring data that can control for sources of variation such as stand structure, type of treatment, and fire weather. The variability in our forests (structure and topography), and in the fire weather that controls fire behavior, generates the need for large samples to achieve statistically significant findings. Even over a 5- to 10-year career of a fuels manager, it would not be typical for more than a handful of treatments with which the manager was involved to be encountered by fire, and the sample would probably not be large enough to support generalization of the findings. Carefully planned, consistently implemented, and adequately funded (over the long-term) monitoring improves the effectiveness and efficiency of the fuels management effort.

\section{Source}

Jain, Theresa B.; Battaglia, Mike A.; Han, Han-Sup; Graham, Russell T.; Keyes, Christopher R.; Fried, Jeremy S.; Sandquist, Jonathan E. 2012. A comprehensive guide to fuel management practices for dry mixed conifer forests in the northwestern United States. Chapter 10. Gen. Tech. Rep. RMRS-GTR-292. Fort Collins, CO: U.S. Department of Agriculture, Forest Service, Rocky Mountain Research Station. 331 p. Available online at www.treesearch.fs.fed.us/pubs/42150 [2012]. For a hard copy, contact Theresa Jain at tjain@fs.fed.us.

\section{Citation}

Jain, Theresa B.; Battaglia, Mike A.; Han, Han-Sup; Graham, Russell T.; Keyes, Christopher R.; Fried, Jeremy S.; Sandquist, Jonathan E. 2014. A comprehensive guide to fuel management practices for dry mixed conifer forests in the northwestern United States: Monitoring. Res. Note RMRSRN-63. Fort Collins, CO: U.S. Department of Agriculture, Forest Service, Rocky Mountain Research Station. 2 p. 